\title{
Efficacy and safety of intravitreal aflibercept in ranibizumab-refractory patients with neovascular age-related macular degeneration
}

\author{
Sam Razavi $1^{*}$, Laurent Kodjikian², Audrey Giocanti-Aurégan ${ }^{3}$, Ingrid Dufour ${ }^{4}$ and Eric Souied ${ }^{5}$
}

\begin{abstract}
Background: Anti-vascular endothelial growth factor (anti-VEGF) agents have become the standard of care in neovascular age-related macular degeneration (nAMD). Despite generally excellent response rates to anti-VEGF therapy, some patients do not respond or may respond suboptimally. In the case of refractory or rapidly recurring fluid in nAMD, clinicians may switch to another anti-VEGF agent. TITAN was an observational study that assessed the effectiveness and safety of intravitreal aflibercept (IVT-AFL) in patients with nAMD refractory to ranibizumab who switched to IVT-AFL after less than 12 months of ranibizumab treatment in routine clinical practice in France.
\end{abstract}

Methods: TITAN was an observational, retrospective and prospective 12-month study conducted at 28 centres in France. Patients with nAMD refractory to ranibizumab were enrolled. Patients who were switched from ranibizumab to IVT-AFL were followed for 12 months. Data were obtained from medical records for retrospectively included patients, and at routine follow-up visits for those included prospectively. The main outcome measure was percentage of patients who achieved treatment success (gain of $\geq 1$ Early Treatment Diabetic Retinopathy Study letters in best-corrected visual acuity [BCVA] and/or any reduction in central retinal thickness [CRT]) from baseline to 12 months after switching. A sample size of 225 patients was determined based on a 2-sided $95 \%$ confidence interval with a width equal to 0.12 when the sample proportion was 0.70 .

Results: We analysed safety data $(N=217)$ and clinical outcomes from patients in the per-protocol population $(n=125)$. The mean (standard deviation) number of IVT-AFL injections was 7.5 (2.6). Treatment success was achieved in $68.8 \%$ of patients. Mean BCVA change from baseline to Month 12 was +1.5 letters $(P=0.105)$ and the mean CRT change was $-45.0 \mu \mathrm{m}(P<0.001)$. In a subgroup analysis, in patients who received three initial monthly IVT-AFL injections, mean BCVA gain was 3.3 letters at Month $12(P=0.015)$. Excluding lack of efficacy and inappropriate scheduling of drug administration, the most common adverse event was eye pain (2.3\%).

Conclusions: Switching ranibizumab-refractory patients with nAMD to IVT-AFL may improve visual outcomes in some patients, particularly those who receive three initial monthly injections.

Trial registration: ClinicalTrials.gov, NCT02321241. First posted: December 22, 2014; Last update posted: July 2, 2018

Keywords: Retina, Age-related macular degeneration, Exudative, Switch, Observational

\footnotetext{
* Correspondence: razavisam3@gmail.com

${ }^{1}$ Centre d'Ophtalmologie, 30 Boulevard Heurteloup, 37000 Tours, France

Full list of author information is available at the end of the article
}

(c) The Author(s). 2021 Open Access This article is licensed under a Creative Commons Attribution 4.0 International License, which permits use, sharing, adaptation, distribution and reproduction in any medium or format, as long as you give appropriate credit to the original author(s) and the source, provide a link to the Creative Commons licence, and indicate if changes were made. The images or other third party material in this article are included in the article's Creative Commons licence, unless indicated otherwise in a credit line to the material. If material is not included in the article's Creative Commons licence and your intended use is not permitted by statutory regulation or exceeds the permitted use, you will need to obtain permission directly from the copyright holder. To view a copy of this licence, visit http://creativecommons.org/licenses/by/4.0/ The Creative Commons Public Domain Dedication waiver (http://creativecommons.org/publicdomain/zero/1.0/) applies to the data made available in this article, unless otherwise stated in a credit line to the data. 


\section{Background}

Neovascular age-related macular degeneration (nAMD) is the most severe form of AMD and is the most common cause of legal blindness [1, 2]. Anti-vascular endothelial growth factor (anti-VEGF) agents, including intravitreal aflibercept (IVT-AFL) and ranibizumab, have become standard of care in nAMD [3-8]. Despite generally excellent response rates to anti-VEGF therapy, some patients do not respond or may respond suboptimally [3-8]. Some studies have shown reduced anatomic response over time with ranibizumab treatment in patients with nAMD, and there have been reports of loss of bioefficacy after repeated ranibizumab treatment [9-11].

In the case of refractory or rapidly recurring fluid in nAMD, clinicians may switch from the current antiVEGF agent to another anti-VEGF agent [12]. The VIEW 1 and VIEW 2 studies [13] assessed the efficacy and safety of IVT-AFL in patients with nAMD and demonstrated non-inferiority of IVT-AFL $2 \mathrm{mg}$, given every 8 weeks after three initial monthly doses, versus ranibizumab $0.5 \mathrm{mg}$, every 4 weeks, in maintaining vision (loss of < 15 Early Treatment Diabetic Retinopathy [ETDRS] letters in best-corrected visual acuity [BCVA]) in treatment-naïve patients over a 12-month period [14-17].

Retrospective studies have shown that individualised IVT-AFL treatment can significantly reduce retinal fluid and preserve vision in patients with nAMD who are resistant to anti-VEGF agents [17-20]. However, prospective studies examining a switch to IVT-AFL in patients refractory to ranibizumab treatment in a real-world setting are scarce [21].

TITAN was an observational study that assessed the effectiveness and safety of IVT-AFL in patients with nAMD refractory to ranibizumab (persistence of intraretinal [IRF] and/or subretinal fluid [SRF]) who switched to IVTAFL after less than 12 months of ranibizumab treatment in routine clinical practice in France.

\section{Methods}

Study design

TITAN (NCT02321241) was an observational 12-month study to assess the effectiveness and safety of IVT-AFL in patients with nAMD refractory to ranibizumab. The study was conducted in 28 centres in France and enrolled patients both retrospectively and prospectively. Data were analysed from patients who received IVTAFL treatment between January 1, 2014 and December 31,2015 . The date of the first IVT-AFL injection was considered the baseline visit, and data collection continued for a maximum of 12 months after the first injection. For retrospectively enrolled patients, the number of office visits, eye exams and treatments were collected from medical records; for prospectively enrolled patients, this information was recorded at routine follow-up visits.
The study protocol was approved by a French data privacy committee (Comité Consultatif sur le Traitement de l'Information en Matière de Recherche dans le Domaine de la Santé and Commission Nationale de l'Informatique et des Libertés). All patients provided written informed consent to participate.

\section{Participants}

Patients diagnosed with nAMD who had been treated with ranibizumab for $>3$ but $<12$ months and switched to prescribed IVT-AFL by their physician were included. Eligible patients must have been refractory to ranibizumab, defined as persistent IRF and/or SRF on optical coherence tomography despite treatment with ranibizumab, in accordance with the Haute Autorite de Santé recommendations of at least three injections of ranibizumab.

Patients excluded from the study were those with any ranibizumab-treated eyes for nAMD previously switched to IVT-AFL, absence of treatment criteria for IVT-AFL, eyes previously treated with photodynamic therapy, another retinal disease (diabetic retinopathy, diabetic macular oedema, myopia or angioid streaks) or participation in any interventional study.

The safety analysis set (SAS) included all patients who received $\geq 1$ IVT-AFL treatment in any eye. The perprotocol (PP) population was defined as all patients in the full analysis set (FAS) (i.e., BCVA $\geq 35$ letters at baseline, or delay of $\leq 380$ days between first and last ranibizumab injection, or $\geq 3$ ranibizumab injections), with BCVA and central retinal thickness (CRT) assessments prior to any treatment (including ranibizumab), at baseline, and at Month 12. The FAS included patients who received $\geq 1$ IVT-AFL treatment and had BCVA and CRT assessments in the study eye at baseline and during follow-up. Patients from the FAS who received a BCVA/ CRT assessment before any treatment (including ranibizumab) at enrolment and at Month 12 were included in the subgroup analysis. This subset of patients was stratified by whether or not they received three initial monthly IVT-AFL injections following the switch.

\section{Outcomes}

The primary endpoint was treatment success rate at 12 months (defined as a gain of $\geq 1$ letter in BCVA and/or any decrease in CRT [in $\mu \mathrm{m}$ ] between initial visit [first injection of IVT-AFL] and 12-month follow-up visit). BCVA was measured using ETDRS letters (preferentially) or any other visual scale. For data analysis, we transformed any other visual acuity score to ETDRS letter score.

Secondary outcomes included change in BCVA between baseline and final study visits (12 months after the first injection of IVT-AFL or study discontinuation), 
mean duration of ranibizumab treatment before initiation of IVT-AFL, and frequency and mean number of IVT-AFL injections over the study period. No BCVA data were collected at the end of ranibizumab treatment; however, switching to IVT-AFL occurred relatively soon after the last ranibizumab injection (median of 44.0 days). Therefore, the change in BCVA during ranibizumab treatment was estimated based on the change between BCVA values before any treatment and before first injection of IVT-AFL.

All adverse events (AEs) occurring after the first IVTAFL injection were documented in the electronic case report form. All patient medical records were evaluated for demographic as well as clinical characteristics, and AEs were summarised using the Medical Dictionary for Regulatory Activities coding system. The event rates for single AEs were calculated based on the total number of documented patients and AEs were categorised according to connection with medication, seriousness, discontinuation of therapy and outcome.

\section{Statistical analyses}

A sample size of 225 patients was determined based on a 2-sided 95\% confidence interval (CI) with a width equal to 0.12 when the sample proportion was 0.70 . Primary analysis criteria (success rate) considered patients who discontinued IVT-AFL treatment prematurely to be treatment failures. We expressed the incidence of treatment success at 12 months as number and percentage of patients (n [\%]) and provided a 2-sided 95\% CI.

The main analysis of secondary criteria (BCVA and CRT) was performed without replacing missing values at study end. Change in BCVA (ETDRS letters) was expressed as mean (standard deviation [SD]) and provided a 2 -sided $95 \%$ CI. We performed two sensitivity analyses with two imputation methods for missing data: imputation of missing value by the last observation carried forward (LOCF) method and imputation of missing data with the median value of population. Statistical analyses were conducted with SAS software release 9.4 (SAS Institute Inc., Cary, NC, USA).

\section{Results \\ Participants}

A total of 236 patients were screened. Of these, 217 were included in the SAS and 125 were included in the PP population (Fig. 1). Demographic characteristics are shown in Table 1.

\section{Ranibizumab treatment and outcomes prior to switching}

Treatment with ranibizumab was initiated soon after diagnosis of nAMD, with a mean lapse of $<1$ month (Table 2). Approximately $75 \%$ of patients had their first injection of ranibizumab $<0.3$ months after diagnosis of nAMD. Patients received a mean (SD) of 5.1 (2.5) injections over 5.6 (4.3) months [22]. Approximately 50\% of patients received $\geq 4$ ranibizumab injections over a median duration of 4.6 months. Mean (SD) BCVA improved significantly with ranibizumab treatment prior to the switch $(+2.2$ [12.0] ETDRS letters, $P=0.046)$ (Table 2). Distribution of patients with SRF, IRF and subretinal pigment epithelium (sub-RPE) at baseline according to the absence or presence of SRF, IRF and sub-RPE before treatment (including ranibizumab), is shown in Fig. 2.

\section{Intravitreal aflibercept treatment and outcomes following the switch}

The success rate (proportion of patients with a gain of $\geq 1$ letter in BCVA and/or any decrease in CRT [in $\mu \mathrm{m}$ ] between baseline [the date of the first IVT-AFL injection] and 12-month follow-up visit) was $68.8 \%$. The mean BCVA improvement from baseline to Month 12 was 1.5 letters $(P=0.105)$ (Table 3$)$. At Month $12,55.2 \%$ of patients $(n=69 / 125)$ were able to read $\geq 70$ letters.

Data on the timing of first injection, treatment duration and number of IVT-AFL injections are shown in

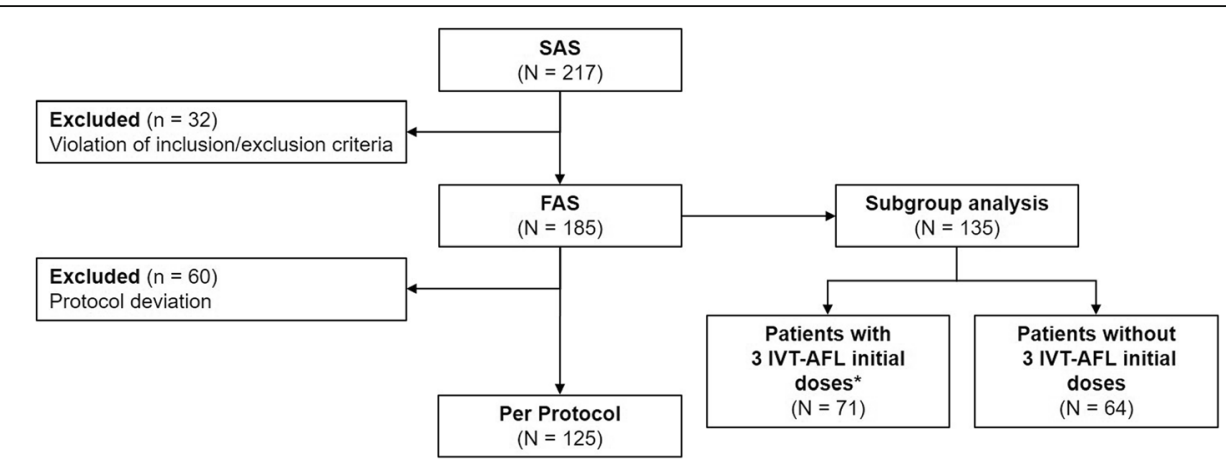

Fig. 1 Patient disposition. *Three initial monthly injections ( $-1 /+2$ weeks). The per-protocol population was defined as patients without protocol deviation and with BCVA/CRT assessments prior to any treatment (ranibizumab) at baseline and at Month $12(n=125)$. The FAS included patients who received $\geq 1$ IVT-AFL treatment and had BCVA and CRT assessments in the study eye at baseline and during follow-up $(n=185)$. BCVA = best-corrected visual acuity; CRT = central retinal thickness; FAS = full analysis set; IVT-AFL = intravitreal aflibercept; SAS = safety analysis set 
Table 1 Demographic Characteristics

\begin{tabular}{|c|c|c|}
\hline & $\begin{array}{l}\text { Full Analysis Set } \\
(n=185)\end{array}$ & $\begin{array}{l}\text { Per-Protocol Set } \\
(n=125)\end{array}$ \\
\hline \multicolumn{3}{|l|}{ Characteristic at baseline } \\
\hline Age, years & $77.6(8.5)$ & $78.2(8.0)$ \\
\hline Female, $n(\%)$ & $109(58.9)$ & $79(63.2)$ \\
\hline Duration of nAMD, months & $8.5(7.3)$ & $7.4(3.3)$ \\
\hline \multicolumn{3}{|c|}{ Characteristic before any treatment, including ranibizumab } \\
\hline BCVA, ETDRS letters & $62.4(16.6)^{\mathrm{a}}$ & $64.0(15.6)$ \\
\hline $\mathrm{CRT}, \mu \mathrm{m}$ & $368.3(124.4)^{b}$ & $362.4(115.2)^{c}$ \\
\hline Subretinal fluid, $n(\%)$ & $133(76.9)^{b}$ & $92(74.2)^{c}$ \\
\hline Intraretinal fluid, $n(\%)$ & $76(43.9)^{b}$ & $55(44.4)^{c}$ \\
\hline Pigment epithelium detachment, $n$ (\%) & $125(72.3)^{b}$ & $88(71.0)^{c}$ \\
\hline
\end{tabular}

Mean (standard deviation) unless otherwise stated

${ }^{\mathrm{a}} n=163 ;{ }^{\mathrm{b}} n=161 ;{ }^{\mathrm{c}} n=116$; values correspond to assessments before any treatment, including ranibizumab, had been administered

BCVA Best-corrected visual acuity, CRT Central retinal thickness, ETDRS Early Treatment Diabetic Retinopathy Study, nAMD Neovascular age-related macular degeneration

Table 3. Given the presence of extreme values, the median data is presented instead of the mean. The delay between the last injection of ranibizumab and the first IVT-AFL injection ranged from 9 to 314 days (mean [SD], 61.2 [46.1]; median 43 days). The main reason for switching from ranibizumab to IVT-AFL was that the patient was considered refractory to ranibizumab.

The range of IVT-AFL treatments received over the 12-month study duration is shown in Fig. 3. More than half of patients $(52.8 \%)$ received three initial monthly IVT-AFL injections. Overall, $17.6 \%(n=22)$ of patients switched at least once from IVT-AFL to ranibizumab, and $5.6 \%(n=7)$ switched back to IVT-AFL.

\section{Anatomic outcomes following the switch}

Anatomic outcomes improved in patients who were refractory to ranibizumab and switched to IVT-AFL (Table 3). Mean CRT reduction from baseline to Month 12 was
$45.0 \mu \mathrm{m}(P<0.001)$. The proportion of patients with SRF at baseline was $70.7 \%(n=87 / 123)$ and at Month 12 was $48.3 \%(n=58 / 120)$. The proportion of patients with IRF was $35.8 \%$ at baseline $(n=44 / 123)$ and $22.5 \%(n=$ $27 / 120)$ at Month 12, respectively. Differences between the proportions of patients with and without fluid at baseline and Month 12 were significant for SRF and IRF (McNemar test $P<0.001$ and $P=0.014$, respectively), but not sub-RPE. Distribution of patients with SRF, IRF and sub-RPE at Month 12 according to the absence or presence of SRF, IRF and sub-RPE at baseline are shown in Fig. 2.

Approximately one-quarter of patients $(24.0 \% ; n=30 /$ 125) gained 0 to 4 letters; $16.8 \%(n=21 / 125)$ gained 5 to 9 letters; $10.4 \%(n=13 / 125)$ gained 10 to 14 letters; and $11.2 \%(n=14 / 125)$ gained $\geq 15$ letters from baseline to Month 12. Among patients gaining $\geq 15$ letters, mean BCVA change was 20.6 (8.0) letters. Conversely, $8.0 \%$

Table 2 Ranibizumab Treatment and Outcomes Before Switch to Intravitreal Aflibercept

\begin{tabular}{ll}
\hline & $\begin{array}{c}\text { Patients } \\
(\boldsymbol{n}=\mathbf{1 2 5})\end{array}$ \\
\hline Delay between diagnosis of nAMD and first injection of ranibizumab, months & $0.5(1.2)$ \\
Number of ranibizumab injections & $4.8(1.9)$ \\
Duration of ranibizumab treatment, months & $4.9(2.8)$ \\
BCVA before any treatment (including ranibizumab), ETDRS letters & $64.0(15.6)$ \\
BCVA at baseline (switch to IVT-AFL), ETDRS letters & $66.2(12.1)$ \\
Change in BCVA from before the start of any treatment (including ranibizumab) and baseline (switch to IVT-AFL) & $2.2(12.0)$ \\
$P$ value & 0.046 \\
\hline
\end{tabular}

All values are expressed as mean (standard deviation); per-protocol population

$P$ value is for paired sample $t$ test

$B C V A$ Best-corrected visual acuity, ETDRS Early Treatment Diabetic Retinopathy Study, IVT-AFL Intravitreal aflibercept, nAMD Neovascular age-related

macular degeneration 


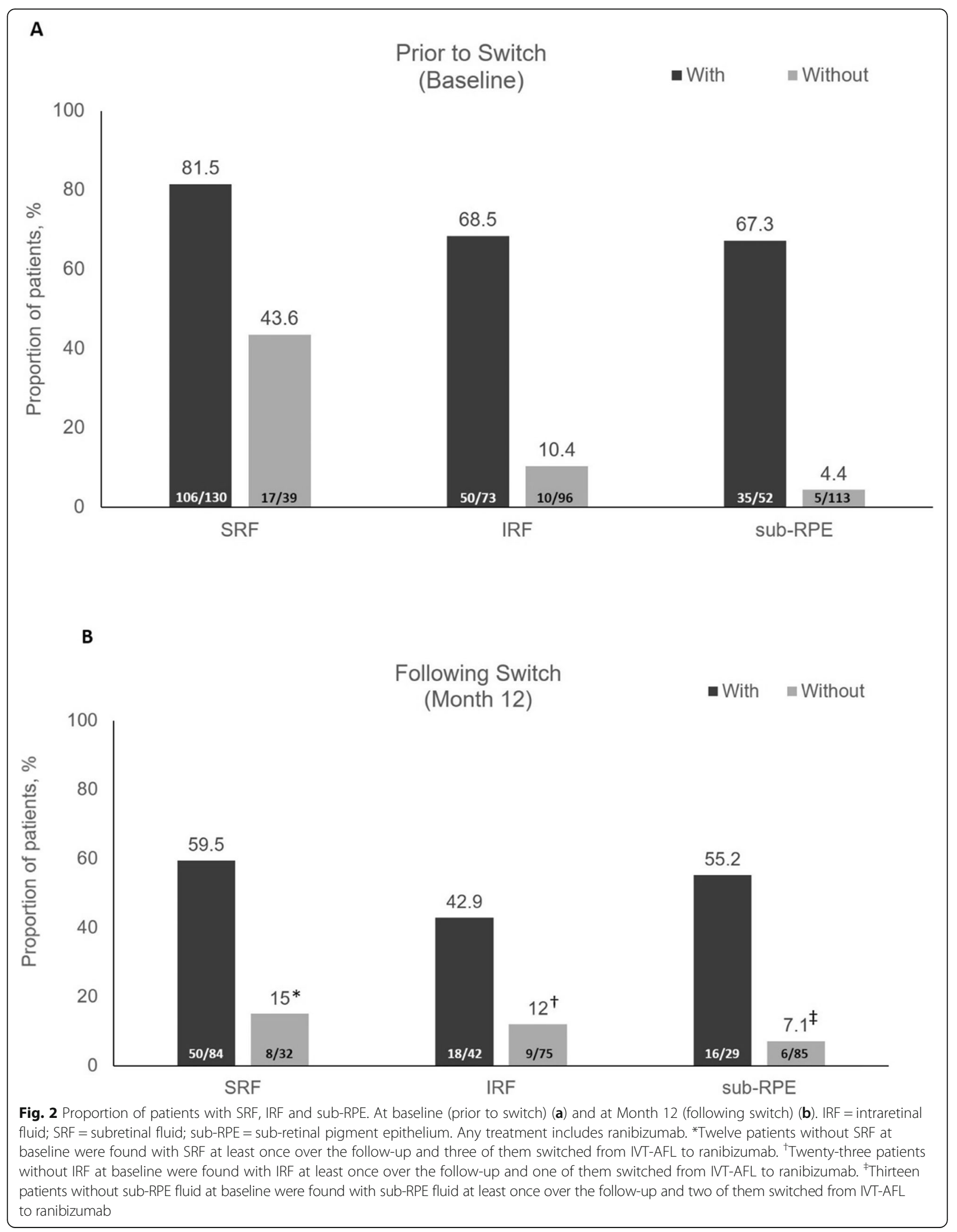


Table 3 Intravitreal Aflibercept Treatment and Outcomes Following Switch to Intravitreal Aflibercept

\begin{tabular}{|c|c|}
\hline & $\begin{array}{l}\text { Patients } \\
(n=125)\end{array}$ \\
\hline Delay between last injection of ranibizumab and first IVT-AFL, days & $61.2(46.1)$ \\
\hline \multicolumn{2}{|l|}{ Reasons for switch to IVT-AFL, $n$ (\%) } \\
\hline Refractory & $122(97.6 \%)$ \\
\hline $\mathrm{AE} / \mathrm{SAE}$ & $3(2.4 \%)$ \\
\hline Other & $0(0 \%)$ \\
\hline Time between diagnosis of nAMD and first IVT-AFL, months & $7.4(3.3)$ \\
\hline Duration of IVT-AFL treatment, months & $11.3(3.1)$ \\
\hline Follow-up duration, months & $12.7(2.0)$ \\
\hline Number of IVT-AFL injections & $7.5(2.6)$ \\
\hline \multicolumn{2}{|l|}{ BCVA, ETDRS letters } \\
\hline Baseline (switch to IVT-AFL) & $66.2(12.1)$ \\
\hline Month 12 & $67.7(13.6)$ \\
\hline Change in BCVA score between baseline and 12 months & $1.5(10.3)$ \\
\hline$P$ value & 0.105 \\
\hline \multicolumn{2}{|l|}{$\mathrm{CRT}, \mu \mathrm{m}$} \\
\hline Baseline (switch to IVT-AFL) & $331.2(103.3)$ \\
\hline Month 12 & $286.2(84.7)$ \\
\hline Change in CRT between baseline and 12 months & $-45.0(101.1)$ \\
\hline$P$ value & $<0.001$ \\
\hline \multicolumn{2}{|l|}{ Patients with SRF, $\boldsymbol{n}(\%)$} \\
\hline Baseline & $87(70.7)$ \\
\hline Month 12 & $58(48.3)$ \\
\hline \multicolumn{2}{|l|}{ Patients with IRF, $\boldsymbol{n}(\%)$} \\
\hline Baseline & $44(35.8)$ \\
\hline Month 12 & $27(22.5)$ \\
\hline
\end{tabular}

All values are reported as mean (standard deviation) unless otherwise indicated; per-protocol population

$P$ values are for the paired samples $t$ test

$A E$ Adverse event, BCVA Best-corrected visual acuity, CRT Central retinal thickness, ETDRS Early Treatment Diabetic Retinopathy Study, IRF Intraretinal fluid;

IVT-AFL Intravitreal aflibercept, nAMD Neovascular age-related macular degeneration, SAE Serious adverse event, SRF Subretinal fluid

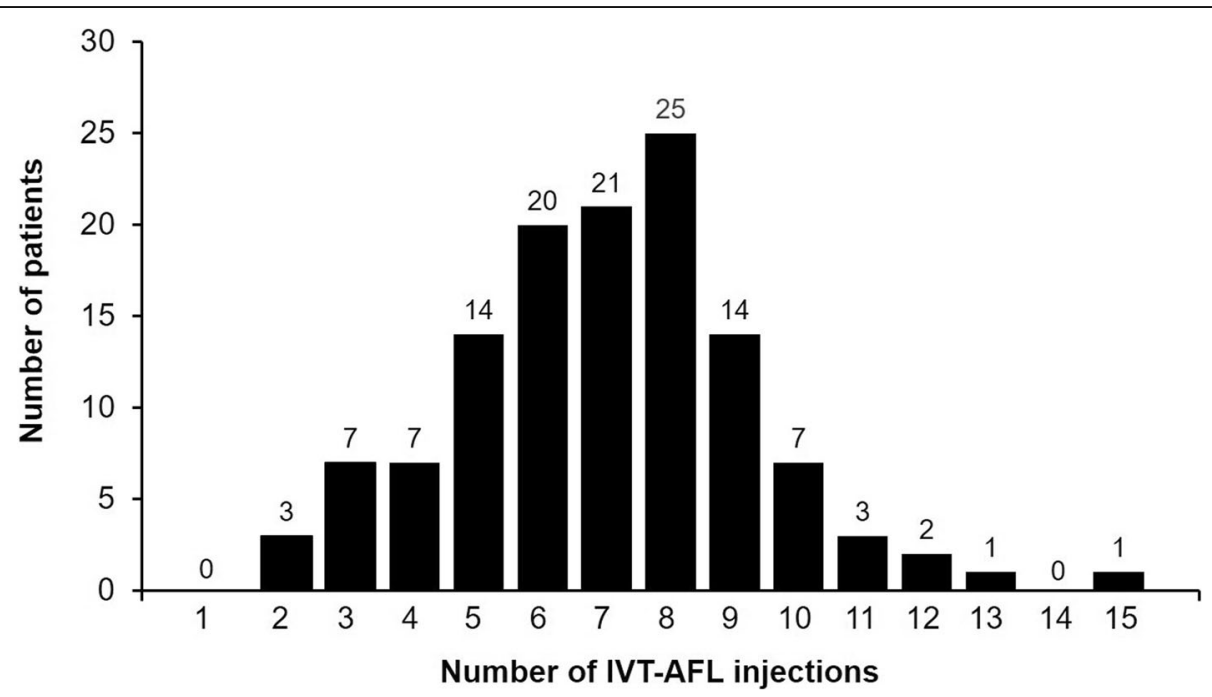

Fig. 3 Distribution of number of intravitreal aflibercept injections over the 12-month study. Per-protocol population. IVT-AFL = intravitreal aflibercept 
( $n=10 / 125)$ lost $\geq 15$ letters. Figure 4 shows the distribution of patients by final absolute BCVA subgroups $(<50$; 50 to $55 ; 55$ to 70 ; and $\geq 70$ letters).

\section{Exploratory and subgroup analyses following switch} In an exploratory analysis conducted in the PP population, patients with $<3$ months of ranibizumab treatment prior to baseline gained a mean (SD) of 4.0 (11.1) letters from baseline to Month $12(P=0.025)$. This gain was greater than that observed in patients with 3 to 5 months, 6 to 8 months, and $\geq 9$ months of ranibizumab treatment prior to baseline $(+0.1[10.8],-0.6[8.2]$ and +2.1 [7.8] letters, respectively) (Supplementary Fig. 1).

In a subgroup analysis in the FAS population (patients with BCVA/CRT assessment before any treatment [including ranibizumab], at baseline and at Month 12), the overall success rate was $66.7 \%$, with higher rates in patients who received three initial monthly IVT-AFL injections compared with those who did not $(69.0 \%$ vs $64.1 \%$, respectively; Table 4). No statistical test was performed, but 95\% CIs largely overlap.

At Month 12, in patients who received three initial monthly IVT-AFL injections, mean BCVA gain was 3.3 letters $(P=0.015)$. Mean BCVA was stable in patients who did not receive three initial monthly injections (Table 4). There was a trend in favour of higher BCVA gains among patients with lower initial BCVA.

\section{Sensitivity analyses}

Results of the sensitivity analyses were consistent with the results of the main analyses. Specifically, the success rate in the overall population was $68.8 \%$ when we replaced missing data by the median of the population, or by LOCF. Mean (SD) gain in BCVA was 1.5 (10.3) letters $(P=0.105)$ when missing data were replaced by the median of the population, or by LOCF. At Month 12, the proportion of patients who could read $\geq 70$ letters was $55.2 \%$ for median value replacement and for LOCF value replacement.

\section{Safety outcomes}

Excluding lack of efficacy and inappropriate scheduling of drug administration, which were also considered AEs according to the protocol and occurred in 12.4 and $5.1 \%$ of patients, respectively, the most common $\mathrm{AE}$ was eye pain (2.3\%) (Table 5). Treatment-emergent serious AEs were reported in $1.8 \%$ of patients, none associated with IVT-AFL treatment. The most common non-ocular AE was falls, occurring in $1.4 \%$ of patients. Twenty-seven patients discontinued IVT-AFL due to lack of efficacy and a further seven discontinued IVT-AFL due to other treatment-emergent AEs; in two patients, the AEs (both PED, one with retinal exudate) were considered related to IVT-AFL.

\section{Discussion}

Results from the TITAN study indicate that approximately two-thirds $(67.7 \%)$ of patients with nAMD who switched from ranibizumab to IVT-AFL treatment (after $\leq 12$ months of treatment with ranibizumab) achieved success, defined as a gain of $\geq 1$ letter in BCVA and/or any decrease in CRT between baseline and Month 12 . Patients considered refractory to first-line treatment with ranibizumab had an additional gain (beyond the mean 2.2 letters initially achieved with ranibizumab) of 1.5 letters at Month 12 after IVT-AFL treatment was initiated, indicating that switching from ranibizumab to IVT-AFL can be considered in ranibizumab-refractory nAMD. There was an inverse relationship between baseline BCVA and visual acuity gain at Month 12 . Generally, patients with lower baseline BCVA had greater visual acuity gains at Month 12 . Patients who had

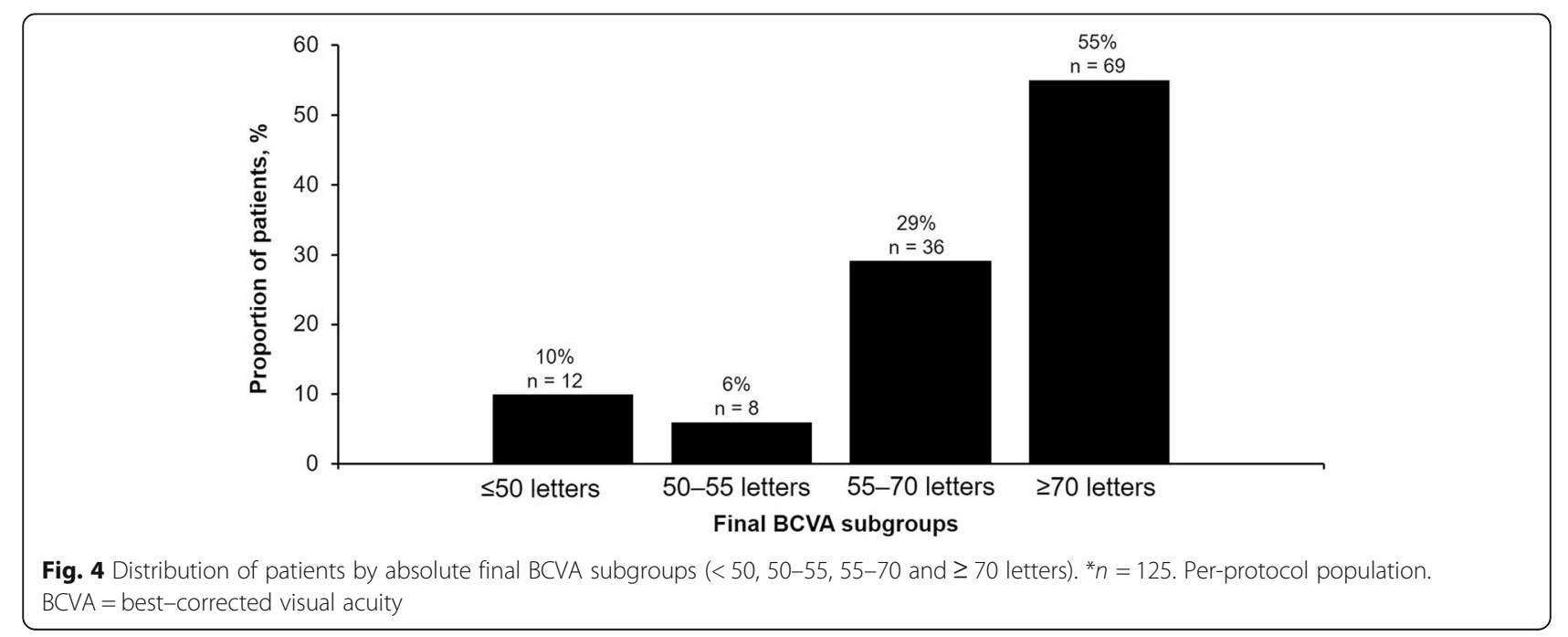


Table 4 Success Rate by Use or Non-use of 3 Initial Monthly Injections (FAS Population)

\begin{tabular}{|c|c|c|c|}
\hline & $\begin{array}{l}\text { Overall } \\
(n=135)\end{array}$ & $\begin{array}{l}\text { With } 3 \text { initial monthly injections } \\
(n=71)\end{array}$ & $\begin{array}{l}\text { Without } 3 \text { initial monthly injections } \\
(n=64)\end{array}$ \\
\hline $\begin{array}{l}\text { Success rate at } 12 \text { months, } \\
n(\%)[95 \% \mathrm{Cl}]\end{array}$ & $\begin{array}{l}90(66.7) \\
{[58.0-74.5]}\end{array}$ & $\begin{array}{l}49(69.0) \\
{[56.9-79.5]}\end{array}$ & $\begin{array}{l}41(64.1) \\
{[51.1-75.7]}\end{array}$ \\
\hline \multicolumn{4}{|l|}{ BCVA, ETDRS letters } \\
\hline Baseline & $65.1(13.7)$ & $63.6(12.3)$ & $66.9(15.1)$ \\
\hline Month 12 & $66.9(15.0)$ & $66.8(12.1)$ & $66.9(17.8)$ \\
\hline Change in BCVA between initial visit and 12 months & $1.7(10.5)$ & $3.3(11.0)$ & $0.0(9.6)$ \\
\hline$P$ value & 0.056 & 0.015 & NS \\
\hline \multicolumn{4}{|l|}{$\mathrm{CRT}, \mu \mathrm{m}$} \\
\hline Baseline & $328.0(100.9)$ & $338.6(114.6)$ & $316.3(82.5)$ \\
\hline Month 12 & $285.3(84.3)$ & $296.1(88.0)$ & $272.6(78.6)$ \\
\hline Change in CRT between initial visit and 12 months & $-47.0(104.1)$ & $-45.5(106.0)$ & $-48.8(102.6)$ \\
\hline$P$ value & $<0.001$ & $<0.001$ & $<0.001$ \\
\hline
\end{tabular}

All values are reported as mean (standard deviation) unless otherwise indicated

$P$ values are for the paired samples $t$ test

${ }^{a}$ Patients who gained $\geq 1$ ETDRS letter (BCVA) and/or a reduction in CRT from baseline (prior to switch) to 12 months after the first intravitreal aflibercept injection BCVA Best-corrected visual acuity, CI Confidence interval, CRT Central retinal thickness, ETDRS Early Treatment Diabetic Retinopathy Study, FAS Full analysis set, NS Not significant

previously received ranibizumab for $<3$ months prior to switch had a greater improvement in BCVA. In addition to decreases in CRT after switching treatment, an enhanced anatomic response was observed, with reductions in fluid accumulation observed in the SRF, IRF and sub-RPE compartments. Overall, these results suggest that considering a switch to IVT-AFL from ranibizumab after $<3$ months may be warranted; however, these findings should be interpreted with caution as the TITAN study was not specifically designed to answer this question, and other confounding variables such as duration of disease are likely to have had an effect.

Findings from other studies of patients refractory to ranibizumab, who switched to IVT-AFL after varying durations of treatment, suggest that there may be a benefit to switching, particularly with anatomical benefit after the switch in terms of improvements in central retinal thickness and pigment epithelium detachment [23]. In fact, the effect of switching on functional outcomes has been shown to be variable [23] and, in our analysis, the overall mean change in BCVA was relatively small. However, over $20 \%$ of the ranibizumab-refractory patients achieved BCVA gains of $\geq 10$ letters after receiving IVT-AFL, suggesting there may be specific subgroups of patients who could respond particularly well to switching to IVT-AFL, despite an initially poor response to anti-VEGF therapy with ranibizumab. Further randomised, controlled studies with appropriate control groups are, however, required.

The present study highlights the importance of three initial monthly injections at the start of IVT-AFL treatment. Greater visual improvements were observed in patients who received three initial monthly injections than in those who did not, which is consistent with previous studies of patients with nAMD who were switched from ranibizumab to IVT-AFL $[21,24]$. It is notable that the proportion of patients who received three initial monthly injections in TITAN was lower $(52.8 \%)$ than expected given the recommended dosing regimen in France [25]. Findings from this study were consistent with the known safety profile of IVT-AFL in nAMD $[13,26]$.

A few limitations inherent in the observational study design should be noted, including the use of different charts to evaluate visual acuity. Here, ETDRS letter charts or any other visual scale were used to evaluate visual acuity; if the latter, results were converted to ETDRS letters, potentially introducing a bias, especially when measuring the number of letters gained or lost after treatment. Also, our study design includes both retrospective and prospective components, and lacks a control group, which is a fundamental aspect of an observational study. In addition, findings are from a single European country, which may not be representative of other countries. Some data were imputed due to variability in data collection; a feature common in observational studies. Given that success was not achieved if patients discontinued IVT-AFL prematurely, even if they switched back to IVT-AFL at a later point, the proportion of patients achieving success may have been underestimated. The success rate was $70.3 \%$ (FAS) using median data. Patients who switched from IVT-AFL prior to receiving 12 months of treatment were considered failures; therefore, replacement of missing data by median value of population only slightly affected the success rate. Finally, factors that could influence differences in visual and anatomic outcomes between groups (e.g., 
disease severity at baseline, frequency of injections and physician monitoring) were not explored in the TITAN study.

\section{Conclusions}

The TITAN study demonstrated the effectiveness and safety of IVT-AFL in patients with ranibizumabrefractory nAMD in routine clinical practice in France. These findings suggest that an early switch $(<12$ months) from ranibizumab to IVT-AFL may improve visual outcomes over 12 months for some patients in this difficult-to-treat population. The study further highlighted that initiating IVT-AFL treatment with three initial monthly injections after switching from ranibizumab may improve visual outcomes. Further studies with appropriate control groups are required to understand how to best identify those patients most likely to benefit from switching to IVT-AFL.

\section{Supplementary Information}

The online version contains supplementary material available at https:/doi. org/10.1186/s12886-021-01841-6.

Additional file 1: Supplementary Figure 1. Visual gains in patients treated with ranibizumab prior to switching to IVT-AFL. Per-protocol population. BCVA = best-corrected visual acuity; IVT-AFL, intravitreal aflibercept.

\section{Abbreviations \\ AE: Adverse event; anti-VEGF: Anti-vascular endothelial growth factor; BCVA: Best-corrected visual acuity; CRT: Central retinal thickness; ETDRS: Early Treatment Diabetic Retinopathy; FAS: Full analysis set; IRF: Intraretinal fluid; IVT-AFL: Intravitreal aflibercept; LOCF: Last observation carried forward; nAMD: Neovascular age-related macular degeneration; PP: Per-protocol; SAS: Safety analysis set; SD: Standard deviation; SRF: Subretinal fluid; sub-RPE: Subretinal pigment epithelium}

\section{Acknowledgements}

The authors wish to thank the TITAN study investigators: Dr. Aouizerate, Dr. Averous, Professor Baillif, Dr. Benouaich, Dr. Benyelles, Professor Berrod, Dr. Cantaloube-Bessière, Dr. Chahed, Professor Chiambaretta, Dr. Cohen, Dr. Coscas, Dr. De Bats, Dr. Deudon-Combe, Dr. Dominguez, Dr. Donati, Professor Dot, Dr. Dumas, Dr. Giocanti-Auregan, Dr. Guigui, Professor Kodjikian, Dr. Oubraham, Dr. Rothschild, Dr. Rumen, Dr. Sampo, Dr. Scholtès, Dr. Sibille-Dabadie, Professor Souied, Dr. Stanescu, Dr. Tran, Dr. Uzzan, Dr. Wolff and Dr. Zerbib. Medical writing assistance was provided by Apothecom, UK, and was funded by Bayer Consumer Care AG, Basel, Switzerland.

\section{Authors' contributions}

All authors have read and approved the manuscript. SR contributed to the design; data acquisition, analysis and interpretation; and preparation and final review of the manuscript. LK contributed to the design; data acquisition, analysis and interpretation; and preparation and final review of the manuscript. AGA contributed to the design; data acquisition, analysis and interpretation; and preparation and final review of the manuscript. ID contributed to the design; data acquisition, analysis and interpretation; and preparation and final review of the manuscript. ES contributed to data acquisition, analysis and interpretation; and preparation and final review of the manuscript.

\section{Funding}

The TITAN study was sponsored by Bayer HealthCare SAS, France. The sponsor participated in the design and conduct of the study, analysis of the data, and preparation of the manuscript.

\section{Availability of data and materials}

Availability of the data underlying this publication will be determined according to Bayer's commitment to the EFPIA/PhRMA "Principles for responsible clinical trial data sharing". This pertains to scope, time point and process of data access.

As such, Bayer commits to sharing, upon request from qualified scientific and medical researchers, patient-level clinical trial data, study-level clinical trial data, and protocols from clinical trials in patients for medicines and indications approved in the United States (US) and European Union (EU) as necessary for conducting legitimate research. This applies to data on new medicines and indications that have been approved by the EU and US regulatory agencies on or after January 1, 2014.

Interested researchers can use www.clinicalstudydatarequest.com to request access to anonymised patient-level data and supporting documents from clinical studies to conduct further research that can help advance medical science or improve patient care. Information on the Bayer criteria for listing studies and other relevant information is provided in the Study sponsors section of the portal.

\section{Ethics approval and consent to participate}

Bayer France received a positive statement from the CCTIRS (Comité Consultatif sur le Traitement de l'Information en Matière de Recherche dans le Domaine de la Santé: Advisory Committee on Information Processing in Material Research in the Field of Health) on 26 November 2014 and an authorization from the CNIL (Commission Nationale de I'Informatique et des Libertés: National Commission on Computer Technology and Freedom) on 24 December 2015 concerning the TITAN study. These gave Bayer the possibility to collect, analyse and use anonymised data of patients included in this study. All patients provided written informed consent to participate.

\section{Consent for publication}

Not applicable.

\section{Competing interests}

SR: Consulting fees from Allergan, Bayer and Novartis; LK: Financial support from Novartis, Allergan, Bayer, Thea and Alcon; consulting fees from Alcon, Alimera, Allergan, Bayer, Roche and Novartis; research funding from Alcon, Alimera, Allergan, Bayer, Horus, Novartis and Thea Pharmaceuticals; AGA: Consulting fees from Alimera, Allergan, Bayer and Novartis; ID: Employee of Bayer HealthCare SAS; ES: Consulting fees and financial support from Allergan, Bayer, Novartis, Roche and Thea Pharmaceuticals.

\section{Author details}

${ }^{1}$ Centre d'Ophtalmologie, 30 Boulevard Heurteloup, 37000 Tours, France. ${ }^{2}$ Centre Hospitalier de la Cr Rousse, Université de Lyon, Lyon, France. ${ }^{3}$ Hôpital Avicenne, AP-HP, Université Paris 13, DHU Vision et Handicaps, Bobigny, France. ${ }^{4}$ Bayer HealthCare SAS, 220 Avenue de la Recherche, 59120 Loos, France. ${ }^{5}$ Centre Hospitalier Intercommunal de Créteil, 40 Verdun avenue, Créteil, 94000 Paris, France.

Received: 7 October 2020 Accepted: 2 February 2021

Published online: 17 February 2021

\section{References}

1. Ohr M, Kaiser PK. Intravitreal aflibercept injection for neovascular (wet) agerelated macular degeneration. Expert Opin Pharmacother. 2012;13(4):58591.

2. Verner-Cole EA, Davis SJ, Lauer AK. Aflibercept for the treatment of neovascular age-related macular degeneration. Drugs Today (Barc). 2012; 48(5):317-29.

3. Rosenfeld PJ, Brown DM, Heier JS, Boyer DS, Kaiser PK, Chung CY, et al. Ranibizumab for neovascular age-related macular degeneration. N Engl J Med. 2006:355(14):1419-31.

4. Brown DM, Michels M, Kaiser PK, Heier JS, Sy JP, lanchulev T. Ranibizumab versus verteporfin photodynamic therapy for neovascular age-related macular degeneration: two-year results of the ANCHOR study. Ophthalmology. 2009;116(1):57-65.e55.

5. Regillo CD, Brown DM, Abraham P, Yue H, lanchulev T, Schneider S, et al. Randomized, double-masked, sham-controlled trial of ranibizumab for neovascular age-related macular degeneration: PIER study year 1. Am J Ophthalmol. 2008;145(2):239-48. 
6. Schmidt-Erfurth U, Eldem B, Guymer R, Korobelnik JF, Schlingemann RO, Axer-Siegel $R$, et al. Efficacy and safety of monthly versus quarterly ranibizumab treatment in neovascular age-related macular degeneration: the EXCITE study. Ophthalmology. 2011;118(5):831-9.

7. Boyer DS, Heier JS, Brown DM, Francom SF, lanchulev T, Rubio RG. A phase IIIb study to evaluate the safety of ranibizumab in subjects with neovascular age-related macular degeneration. Ophthalmology. 2009;116(9):1731-9.

8. Busbee BG, Ho AC, Brown DM, Heier JS, Suner IJ, Li Z, et al. Twelve-month efficacy and safety of $0.5 \mathrm{mg}$ or $2.0 \mathrm{mg}$ ranibizumab in patients with subfoveal neovascular age-related macular degeneration. Ophthalmology. 2013;120(5):1046-56

9. Binder S. Loss of reactivity in intravitreal anti-VEGF therapy: tachyphylaxis or tolerance? Br J Ophthalmol. 2012;96(1):1-2.

10. Eghoj MS, Sorensen TL. Tachyphylaxis during treatment of exudative age-related macular degeneration with ranibizumab. Br J Ophthalmol. 2012;96(1):21-3.

11. Schaal S, Kaplan HJ, Tezel TH. Is there tachyphylaxis to intravitreal antivascular endothelial growth factor pharmacotherapy in age-related macular degeneration? Ophthalmology. 2008;115(12):2199-205.

12. Papadopoulos N, Martin J, Ruan Q, Rafique A, Rosconi MP, Shi E, et al. Binding and neutralization of vascular endothelial growth factor (VEGF) and related ligands by VEGF trap, ranibizumab and bevacizumab. Angiogenesis. 2012:15(2):171-85

13. Heier JS, Brown DM, Chong V, Korobelnik JF, Kaiser PK, Nguyen QD, et al. Intravitreal aflibercept (VEGF trap-eye) in wet age-related macular degeneration. Ophthalmology. 2012;119(12):2537-48.

14. Gasperini JL, Fawzi AA, Khondkaryan A, Lam L, Chong LP, Eliott D, et al. Bevacizumab and ranibizumab tachyphylaxis in the treatment of choroidal neovascularisation. Br J Ophthalmol. 2012;96(1):14-20.

15. Blanco-Garavito R, Jung C, Uzzan J, Quaranta-EIMaftouhi M, Coscas F, Sahel $J$, et al. Aflibercept after ranibizumab intravitreal injections in exudative agerelated macular degeneration: the ARI2 study. Retina. 2018;38(12):2285-92.

16. Stewart MW. Clinical and differential utility of VEGF inhibitors in wet agerelated macular degeneration: focus on aflibercept. Clin Ophthalmol. 2012;6: 1175-86.

17. Yonekawa Y, Andreoli C, Miller JB, Loewenstein JI, Sobrin L, Eliott D, et al. Conversion to aflibercept for chronic refractory or recurrent neovascular age-related macular degeneration. Am J Ophthalmol. 2013;156(1):29-35 e22

18. Kumar N, Marsiglia M, Mrejen S, Fung AT, Slakter J, Sorenson J, et al. Visual and anatomical outcomes of intravitreal aflibercept in eyes with persistent subfoveal fluid despite previous treatments with ranibizumab in patients with neovascular age-related macular degeneration. Retina. 2013;33(8):1605-12.

19. Gharbiya M, lannetti L, Parisi F, De Vico U, Mungo ML, Marenco M. Visual and anatomical outcomes of intravitreal aflibercept for treatment-resistant neovascular age-related macular degeneration. Biomed Res Int. 2014;2014: 273754

20. Messenger WB, Campbell JP, Faridi A, Shippey L, Bailey ST, Lauer AK, et al. Injection frequency and anatomic outcomes 1 year following conversion to aflibercept in patients with neovascular age-related macular degeneration. Br J Ophthalmol. 2014;98(9):1205-7.

21. Chang AA, Li H, Broadhead GK, Hong T, Schlub TE, Wijeyakumar W, et al. Intravitreal aflibercept for treatment-resistant neovascular age-related macular degeneration. Ophthalmology. 2014;121(1):188-92.

22. Lucentis [prescribing information]. South San Francisco: Genentech, Inc.; 2018.

23. Empeslidis T, Storey M, Giannopoulos T, Konidaris V, Tranos PG, Panagiotou $E S$, et al. How successful is switching from bevacizumab or ranibizumab to aflibercept in age-related macular degeneration? A systematic overview. Adv Ther. 2019;36(7):1532-48.

24. Aghdam KA, Pielen A, Framme C, Junker B. Visual and anatomic outcomes after conversion to aflibercept in neovascular age-related macular degeneration: 12-month results. Eur J Ophthalmol. 2016;26(5):473-8.

25. Eylea [prescribing information]. Tarrytown: Regeneron Pharmaceuticals, Inc.; 2018.

26. Schmidt-Erfurth U, Kaiser PK, Korobelnik JF, Brown DM, Chong V, Nguyen $\mathrm{QD}$, et al. Intravitreal aflibercept injection for neovascular age-related macular degeneration: ninety-six-week results of the VIEW studies. Ophthalmology. 2014;121(1):193-201.

\section{Publisher's Note}

Springer Nature remains neutral with regard to jurisdictional claims in published maps and institutional affiliations.

\section{Ready to submit your research? Choose BMC and benefit from:}

- fast, convenient online submission

- thorough peer review by experienced researchers in your field

- rapid publication on acceptance

- support for research data, including large and complex data types

- gold Open Access which fosters wider collaboration and increased citations

- maximum visibility for your research: over $100 \mathrm{M}$ website views per year

At BMC, research is always in progress.

Learn more biomedcentral.com/submissions 